\title{
Postoperative Complications of Minimally Invasive Plate Osteosynthesis (MIPO) for Distal Tibial Metaphyseal Fractures
}

\author{
Kap Jung Kim, Sang Ki Lee, Hwa Yeol Yang, Won-Sik Choy \\ Department of Orthopaedic Surgery, Eulji University College of Medicine, \\ 1306 Dunsan-dong, Seo-gu, Daejeon(322-799), Republic of Korea \\ Correspondent Author: Kap Jung Kim (E-mail : oskkj@eulji.ac.kr)
}

\begin{abstract}
The minimally invasive plate osteosynthesis (MIPO) technique is a widely used surgical option for treating distal tibial methphyseal fractures. The purpose of this study was to analyze results of the MIPO technique for distal tibial metaphyseal fractures and postoperative complications. Sixty-one patients with distal tibial metaphyseal fractures were included. They were followed for more than 12 months. Mean age was 53.1 years (range, 18-85 years). Males were 27 cases and females were 34 cases. Fractures were classified according to the AO/OTA classification system. Postoperative complications, radiological results, and clinical results were analyzed. The mean follow-up period was 61.9 months (range, 12-103). According to the AO/OTA classification, 11 cases were A1, 16 were A2, 18 were $A 3,10$ were B1, and 6 were B2. The mean time to bony union was 13.8 weeks (range, 12-24 weeks). Seven postoperative complications occurred in six patients including two infections, three overlying skin necrosis, and two nonunions. Fiftysix patients (91.8\%) had excellent and good results and five (8.2\%) had fair results. The MIPO technique was an effective surgical option for distal tibial metaphyseal fractures that produced good clinical and radiological results. Although the MIPO technique has many advantages, care should be taken for possible postoperative complications that may require additional surgery.
\end{abstract}

Keywords: Distal tibia, Fractures, $M I P O$

\section{Introduction}

Distal tibial metaphyseal fractures are common long bone fractures and are more complicated to treat than diaphyseal fractures. Non-operative treatment of these fractures results in unacceptable deformities and ankle stiffness [1]. Distal tibial metaphyseal fractures are very close to the ankle joint and are commonly associated with severe communion and extensive soft tissue injury [2]. Various treatment options have been used, including intramedullary nailing, plating, and external fixation. Intramedullary nailing is useful to treat a tibial diaphyseal fracture, however, the risk of fracture propagation into the ankle joint or a failed nail due to inadequate hold of the small distal tibial fracture fragment can lead to malalignment. Conventional open reduction and internal fixation with a plate achieves good reduction, rigid fixation and permits early mobilization. However, this technique requires extensive exposure and results in many complications, such as infection, wound breakdown, nonunion, or delayed union [3-6]. Surgical dissection to achieve anatomical reduction evacuates an osteogenic fracture hematoma and can strip soft tissue. To minimize the risks of complications, minimally invasive percutaneous osteosynthesis (MIPO) has been a favored technique without making an incision at the fracture site. The MIPO technique preserves the soft tissue envelope and the periosteum, as well as maintains the arterial blood supply. Therefore this procedure reduces surgical trauma to the injury zone. A high success rate and good clinical results from the MIPO technique have been reported in patients with distal tibial metaphyseal 
fractures $[2,7-8]$. Although this technique has many advantages, several complications have been reported, such as skin irritation overlying the plates, skin necrosis, and plate exposure [2, 9-12]. Little has been reported about postoperative complications of the MIPO technique in patients with distal tibial metaphyseal fractures. Therefore, in this study, we discussed postoperative complications of the MIPO technique, with special attention to the details of the complications. Furthermore, we determined whether the MIPO technique remains a good surgical option for treating distal tibial metaphyseal fractures.

\section{Materials and Methods}

The study was performed with the approval of the institutional review of board. Sixtyone patients with distal tibial metaphyseal fractures were included in this study. The absolute indications for surgery were displaced and unstable fractures. Pediatric fractures, pathological fractures, lower limb fractures in patients with a pre-existing neurologic deficit, and those with autoimmune diseases were excluded from the study. All patients were followed for more than 12 months. Mean age was 53.1 years (range, 18-85 years). Males were 27 cases and females were 34 cases. Fractures were classified according to the AO/OTA classification system; 11 cases were A1, 16 cases were A2, 18 cases were A3, 10 cases were B1, and 6 cases were B2. Concurrent fibular fractures occurred in 52 cases (85.2\%) (Table 1).

The patient was placed in the supine position on a radiolucent table under general or spinal anesthesia. Intra-operative fluoroscopy was used throughout the procedure. Both the injured and non-injured limbs were draped above the knee to allow alignment to be checked against the uninjured limb. The fibular fracture was fixed first in cases of concurrent fibular fractures. Then, the distal tibial fracture was indirectly reduced by manual traction under $\mathrm{C}$-arm fluoroscopy. The main fragments of the tibial fracture were aligned and reduced percutaneously. Large fragments were reduced with individual Kirschner wires. If appropriate length and rotation could be achieved, a plate was inserted to bridge the fracture between the fascia and soft tissue along the anteromedial surface of the tibia.

Periodic radiological results were assessed to evaluate bony union. Bony union was defined when a callus formed on more than $75 \%$ of the main fracture line on anteroposterior and lateral radiographs, and no motion or tenderness was detected at the fracture site. Clinical results were analyzed using the IOWA-ankle rating system [13]. Postoperative complications were also recorded and analyzed.

Table 1. Patient Demographic Data

\begin{tabular}{c|c}
\hline Parameter & Value \\
\hline Mean age (years) & 53.1 (range, 18 85) \\
Gender & $27(44 \%)$ \\
Male & $34(56 \%)$ \\
Female & $61.9($ range, $12 \sim 103)$ \\
Mean follow-up (months) & $11(18 \%)$ \\
AO/OTA classification & $16(26.3 \%)$ \\
A1 & $18(29.5 \%)$ \\
A2 & $10(16.4 \%)$ \\
A3 & $6(9.8 \%)$ \\
B1 & $52(85.2 \%)$ \\
B2 & $13.8($ range, $12 \sim 24)$
\end{tabular}




\begin{tabular}{c|c} 
IOWA-ankle rating scale & \\
Excellent & $23(37.7 \%)$ \\
Good & $33(54.1 \%)$ \\
Fair & $5(8.2 \%)$ \\
Postoperative complications & 7 \\
Infection & $2(3.3 \%)$ \\
Skin necrosis & $3(4.9 \%)$ \\
Nonunion & $2(3.3 \%)$ \\
\hline
\end{tabular}

\section{Results}

\subsection{General Results}

All patients were treated using metaphyseal plates and the MIPO technique. Forty fibular fractures $(76.9 \%)$ of the total 52 concurrent fibular fractures were fixed simultaneously. The mean follow-up period was 61.9 months (range, 12-103 months). Radiological evidence of bony union was obtained in all cases except one patient with diabetes. Mean time to bone union was 13.8 weeks (range, 12-24 weeks). The clinical results using the IOWA-ankle rating system ${ }^{13)}$ were as follows: excellent in 23 patients $(37.7 \%)$, good in $33(54.1 \%)$, and fair results in 5 patients $(8.2 \%)$. Fifty-six patients $(91.8 \%)$ had excellent or good results (Table 1).

\subsection{Postoperative Complications}

Seven postoperative complications occurred in six patients: two infections, three overlying skin necrosis, and two nonunions. Two complications (infection and nonunion) occurred in one patient with diabetes (Table 2). The first infection case was treated successfully with a course of antibiotics and debridement. The second case of infection was deep seated and difficult-to-treat, but subsided after the plate was removed. We experienced three cases of overlying skin necrosis. One case was treated with vacuum assisted closure (VAC) following debridement of necrotic skin. Two cases of skin necrosis were too large to be covered nonoperatively. Thus, flap surgeries were performed to cover the soft tissue defect. An autologous bone graft was used in the first case of nonunion; however, bone union was not achieved in the second case, which was combined with infection.

Table 2 Details of the Postoperative Complications

\begin{tabular}{cccc}
\hline No. & Age/sex & Complication & Additional procedures \\
\hline 1 & $46 / \mathrm{F}$ & Nonunion & Autologous bone graft \\
2 & $26 / \mathrm{M}$ & Skin necrosis & Vacuum assisted closure (VAC) \\
3 & $73 / \mathrm{F}$ & Skin necrosis & Flap surgery \\
4 & $51 / \mathrm{F}$ & Skin necrosis & Flap surgery \\
5 & $31 / \mathrm{M}$ & Infection & Incision and debridement \\
6 & $51 / \mathrm{M}$ & Infection & Hardware removal, External fixation \\
& & Nonunion & - \\
\hline
\end{tabular}




\section{Discussion}

Distal tibial metaphyseal fractures are difficult to treat due to poor soft tissue coverage and are often comminuted with small metaphyseal fragments [14]. Traditional techniques may further disrupt the already damaged soft tissue envelope [15]. The concept of bridging plate osteosynthesis has been used to treat multi-fragmentary long bone shaft fractures. This technique minimizes soft tissue injury, which theoretically preserves a better blood supply around the fracture site [16]. Therefore, more rapid fracture union can be achieved due to less soft tissue damage and the preserved blood supply.

Borrelli et al [16] analyzed the extraosseous blood supply of the tibia in a human cadaveric study. The anatomic network of arteries, including the anterior tibial artery and the posterior tibial artery form the extraosseous blood supply of the medial side of the distal tibia [16].

Conventional open plating at the medial aspect of the distal tibia can lead to greater disruption of the extraosseous blood supply than when using percutaneously applied plates [16]. Open plating prevents filling of each periosteal vessel in the region compared to that of percutaneous plates. Disrupting the extraosseous blood supply following fracture might increase the risk of delayed union or nonunion. These findings support efforts to apply less invasive methods to stabilize distal tibial fractures.

We used the metaphyseal locking compression plate (LCP) or periarticular locking plate in all cases in an attempt to minimize the discomfort associated with bulky plates, such as the conventional dynamic compression plate (DCP), [2, 17-18] or the limited bone contact-DCP (LC-DCP) [7, 10, 19]. Use of a low profile LCP or periarticular locking plate along the anteromedial surface of the tibia results in better cosmesis and less chance of impingement. However, 24 patients (40\%) complained of impingement, particularly over the proximal end of the plate. This symptom was tolerable and disappeared immediately after removing the implant. Three cases of overlying skin necrosis with exposed plates occurred at distal end of the plate. As mentioned above, one case was solved with vacuum assisted closure (VAC) following debridement of necrotic skin. Flap surgery was performed in two cases with large areas of skin necrosis (Figure 1). We assumed two probable mechanisms for the overlying skin necrosis at the distal end of plate. First, blood supply to the soft tissue envelope may have been disrupted, even though we used low profile plates and the MIPO technique. Second, the extraosseous blood supply to the distal tibia may have been damaged at the time of fracture. Thus, the soft tissue envelope was vulnerable to poor blood supply, which could have resulted in the overlying skin necrosis.

Postoperative infection is an important complication. The rate of infection following plating is $0-6 \%[2,7,10,17,19-21]$. The range varies because the degree of soft tissue injury differed among the studies. A superficial infection can be successfully treated with antibiotics or abscess drainage. However, a postoperative infection occurs in a patient with an underlying medical illness, it is quite difficult to control and could disseminate into a deep-seated infection. Our second infection case occurred in a 51-year-old male who had uncontrolled diabetes. He developed acute postoperative signs of infection with an abscess and a discharging sinus 1 week postoperatively. C-reactive protein level increased to $15 \mathrm{mg} / \mathrm{dl}$ combined with septicemia. A swab culture from the second case of infection revealed methicillin-sensitive Staphylococcus aureus (MRSA). This was the only case in which the plate removal was needed to control the infection and, he was converted to an external fixator. After controlling the infection, we performed the MIPO technique again to manage the fracture, but bony union was not achieved. We had no positive bacteriological culture from the first infection case, suggesting more easily controllable superficial infection. However, an infection with abscess requires more aggressive treatment in a patient with an underlying medical illness. 


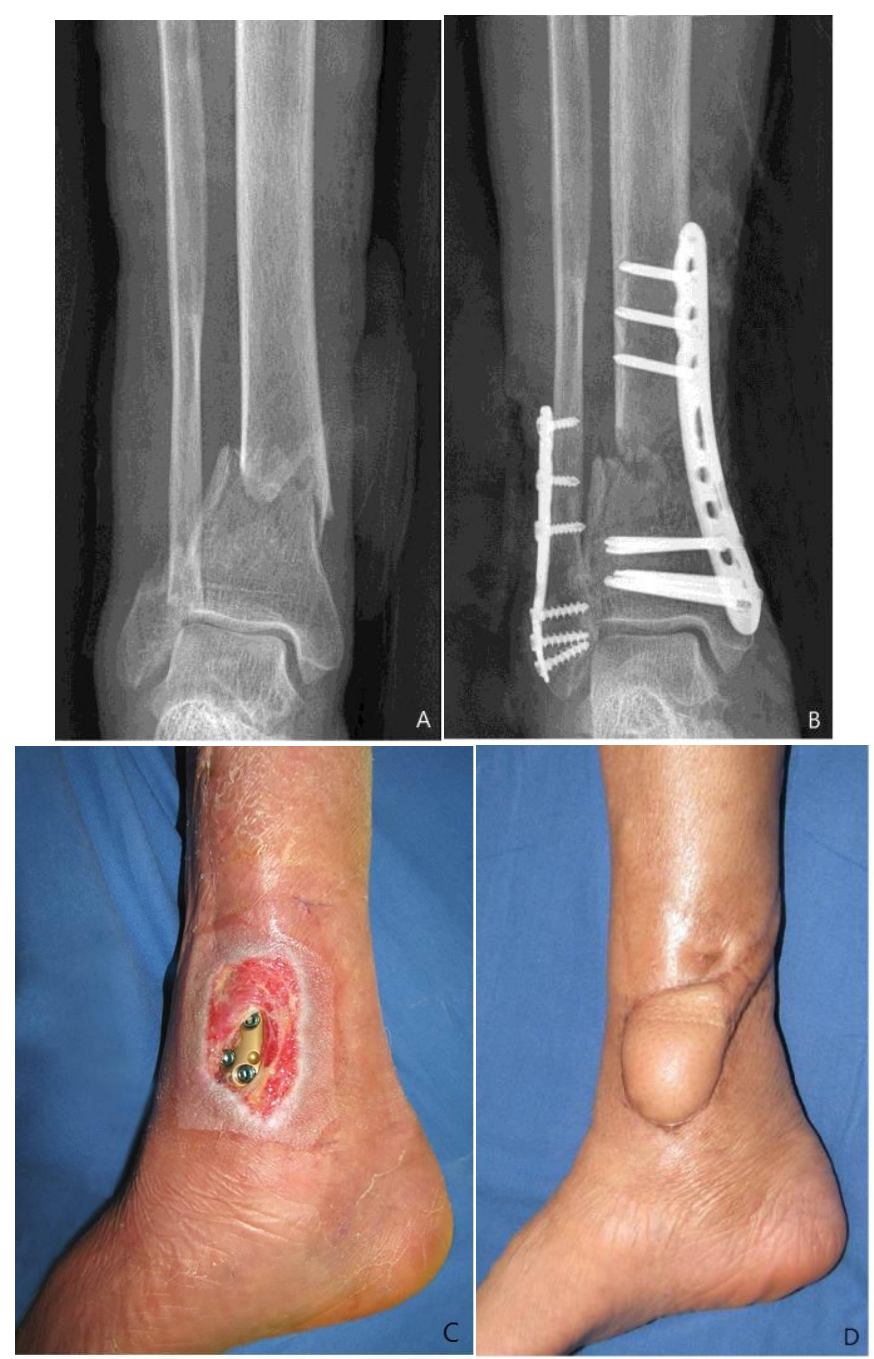

Figure 1 (A) Anteroposterior Radiograph of a 73-year-old Female who Sustained a Distal Tibial Metaphyseal Fracture and a Concurrent Fibular

Fracture. (B) The Minimally Invasive Plate Osteosynthesis (MIPO)

Technique was used on the Anteromedial Side of the Tibia to Treat the distal Tibial Fracture. (C) Photograph Showing Overlying Skin Necrosis and Exposure of the Distal End of the Plate. (D) Photograph Showing Sural Flap to Cover Skin Necrosis

\section{Conclusion}

The MIPO technique is an effective and safe surgical option for treating distal tibial metaphyseal fractures compared with other surgical options. It also provided satisfactory clinical and radiological results. Although MIPO technique has many advantages, care should be taken for possible postoperative complications when using this technique, as additional surgery may be required in some cases.

\section{Acknowledgements}

No benefits in any form have been received or will be received from a commercial party related directly or indirectly to the subject of this article. 


\section{References}

[1] J. M. Digby, G. M. Holloway and J. K.Webb, “A study of function after tibial cast bracing. Injury, vol. 14, (1983), pp 432-439.

[2] D. J. Redfern, Syed SU, Davies SJ. Fractures of the distal tibia: minimally invasive plate Osteosynthesis. Injury. vol. 35, (2004), pp 615-620.

[3] S. M. Teeny, D. A. Wiss, "Open reduction and internal fixation of tibial plafond fractures. Variables contributing to poor results and complications. Clin Orthop Relat Res, vol. 292, (1993), pp 108-117.

[4] W. D. Fisher, D. L. Hamblen, "Problems and pitfalls of compression fixation of long bone fractures: a review of results and complications. Injury. vol. 10, (1978), pp 99-107.

[5] O. Karlström, "Tibial fractures treated by AO compression Osteosynthesis. Experiences from a five year material. Acta Orthop Scand Suppl. vol. 140, (1972), pp 1-104.

[6] T. P. Rüedi, Allgöwer M, "The operative treatment of intra-articular fractures of the lower end of the tibia. Clin Orthop Relat Res. vol. 138, pp 105-110, (1979).

[7] T. Borg, S. Larsson, U Lindsjö, "Percutaneous plating of distal tibial fractures. Preliminary results in 21 patients. Injury, vol. 35, (2004), pp. 608-614.

[8] S. Ghera, F. S. Santori, M. Calderaro and T. L. Giorgini, "Minimally invasive plate Osteosynthesis in distal tibial fractures: pitfalls and surgical guidelines. Orthopedics, vol. 27, (2004), pp. 903-905.

[9] D. L. Helfet, M. Suk, "Minimally invasive percutaneous plate Osteosynthesis of fractures of the distal tibia. Intr Course Lect. vol. 53, (2004), pp. 471-475.

[10] C. W. Oh, H. S. Kyung, I. H. Park, P. T. Kim, Ihn JC. Distal tibia metaphyseal fractures treated by percutaneous plate osteosynthesis. Cln Orthop Relat Res. vol. 408, (2003), pp. 286-291.

[11] J. W. Kim, C. W. Oh, W. J. Jung, J. S. Kim, "Minimally invasive plate osteosynthesis for open fractures of the proximal tibia. Clin Orthop Surg. vol. 4, (2012), pp. 313-320.

[12] J. Park, S. Y. Jeong, "Complications and outcomes of minimally invasive percutaneous plating for proximal humeral fractures. Clin Orthop Surg. vol. 6, (2014), pp. 146-152.

[13] T. C. Merchant, F. R. Dietz, "Long-term follow-up after fractures of the tibial and fibular shafts. J Bone Joint Surg Am. vol. 71, (1989), pp. 599-606.

[14] D. B. Thordarson, "Complications after treatment of tibial pilon fractures: prevention and management strategies. J Am Acad Orthop Surg. vol. 8, (1989), pp. 253-265.

[15] S. K. Bonar, J. L. Marsh, "Tibial plafond fractures: changing principles of treatment. J Am Acad Orthop Surg. vol. 2, (1994), pp. 297-305.

[16] J. Borrelli Jr., W. Prickett, E. Song, D. Becker and W. Ricci, "Extraosseous blood supply of the tibia and the effects of different plating techniques: a human cadaveric study. J Orthop Trauma. vol. 16, (2002), pp. 691-695.

[17] N. Maffulli, A. D. Toms, A. McMurtie, F. Oliva, "Percutaneous plating of distal tibial fractures. Int Orthop, vol. 28, (2004), pp. 159-162.

[18] C. Ryf, U. Götsch, T. Perren, P. Rillmann, "New surgical treatment procedures in fractures of the distal tibia (LCP, MIPO). Ther Umsch, vol. 60, (2003), pp. 768-775.

[19] T. Krackhardt, J.Dilger, I. Flesch, D. Höntzsch, C. Eingartner and K. Weise, "Fractures of the distal tibia treated with closed reduction and minimally invasive plating. Arch Orthop Trauma Surg, vol. 125, (2005), pp. 87-94.

[20] A. D. Toms, A. McMurtie, N. Maffulli, "Percutaneous plating of the distal tibia. J Foot Ankle Surg. vol. 43, (2004), pp. 199-203.

[21] B. A. Zelle, M. Bhandari, M. Espiritu, K. J. Koval, Zlowodzki, "Evidence-Based Orthopaedic Trauma Working Group. Treatment of distal tibia fractures without articular involvement: a systemic review of 1125 fractures. J Orthop Trauma, vol. 20, (2006), pp. 76-79. 